\title{
Role of Early Neuroimaging in Managing Acute Isolated Ocular Motor Nerve Palsies
}

\author{
Tal Paz ${ }^{1, ~ *, ~ N i v ~ L e v y ~}{ }^{1}$, Hana Leiba ${ }^{1,2}$, Daniel Rappoport ${ }^{1,2}$ \\ ${ }^{1}$ Ophthalmology Department, Kaplan Medical Center, Rehovot, Israel \\ ${ }^{2}$ Hadassah and Hebrew University Medical School, Jerusalem, Israel
}

Email address:

dr.talpaz@gmail.com (T. Paz)

${ }^{*}$ Corresponding author

\section{To cite this article:}

Tal Paz, Niv Levy, Hana Leiba, Daniel Rappoport. Role of Early Neuroimaging in Managing Acute Isolated Ocular Motor Nerve Palsies. International Journal of Ophthalmology \& Visual Science. Vol. 4, No. 1, 2019, pp. 24-29. doi: 10.11648/j.ijovs.20190401.15

Received: April 28, 2019; Accepted: May 30, 2019; Published: June 11, 2019

\begin{abstract}
Background: The role of early neuroimaging in older vasculopathic patients presenting with acute isolated ocular motor nerve palsy is still being debated. We wanted to demonstrate the approach differences between neurologists and ophthalmologists, and examine the role of early neuroimaging in managing acute ocular motor mononeuropathies. Methods: Retrospective chart review. Patients $\geq 50$ years with vasculopathic risk factors, presenting with acute isolated third, fourth or sixth cranial nerve palsies. We compared the rate of early neuroimaging referral between neurologists and ophthalmologists at initial presentation, and assessed the proportion of cases in which the final diagnosis has changed after early neuroimaging, in a single medical center. Results: 54 patients were included. After excluding patients with third nerve palsy, the rate of patients referred to early neuroimaging was significantly greater when initially presented to a neurologist compared with an ophthalmologist (29/38 patients were referred to early neuroimaging, of whom twenty by a neurologist and nine by an ophthalmologist, $p<0.001)$. Out of 38 patients presented with fourth or sixth nerve palsies, only $4 / 38(10 \%)$ were found to have a cause other than presumed microvascular ischemia, and only $2 / 29(7 \%)$ patients referred to neuroimaging were found to have a causative lesion. Conclusions: The decision to perform early neuroimaging in older patients with acute isolated $4^{\text {th }}$ or $6^{\text {th }}$ nerve palsies and vasculopathic risk factors could be weighed against observation alone. Thorough history taking and prudent physical examination are important for identifying patients with greater risks, therefore needing early neuroimaging.
\end{abstract}

Keywords: Neuroimaging, Magnetic Resonance Imaging (MRI), Cranial Nerve, Palsy, Acute, Isolated

\section{Introduction}

Isolated third, fourth and sixth cranial nerve palsies are caused by various etiologies. In patients older than 50 years of age, the most common cause is presumed microvascular ischemia in the setting of atherosclerotic risk factors, such as diabetes mellitus, hypertension and hyperlipidemia [1-3]. Spontaneous resolution occurs in $70 \%$ of these cases [3], typically within a period of approximately three months [24]. With increasing use of magnetic resonance imaging (MRI) over the past 20 years, there have been several published reports of other causes of acute ocular motor mononeuropathies, including demyelinating disease, pituitary apoplexy and brainstem infarction/hemorrhage [1, 5-33].

Although neuroimaging is generally considered necessary in all individuals presenting with third nerve palsies, the role of early neuroimaging in older individuals (age $\geq 50$ years) with vasculopathic risk factors presenting with acute isolated $4^{\text {th }}$ or $6^{\text {th }}$ nerve palsy is still under debate and there is a lack of consensus between different medical specialties. In prior prospective studies that have addressed this matter, a percentage of $1-15 \%$ of patients had non-microvascular causes of acute ocular motor mononeuropathies [5-7].

A recent prospective study [1] found that overall, $16.5 \%$ of 109 adult patients presenting with acute ocular motor nerve palsy had structural lesions on MRI scans. In this study, among 80 patients with vasculopathic risk factors alone, $10 \%$ had other causes explaining their ocular motor nerve palsy. After exclusion of patients with third nerve palsies and giant cell arteritis, it was found there is approximately 5\% chance that a patient with vasculopathic risk factors alone, 
presenting with $4^{\text {th }}$ or $6^{\text {th }}$ cranial nerve palsy, had another cause found for the mononeuropathy [1]. According to this, some authors are in favor of performing early neuroimaging during the initial evaluation of adults older than 50 years of age with vasculopathic risk factors, presenting with acute ocular motor mononeuropathies [5-6].

On the other hand, other studies recommend observation alone without early neuroimaging, as long as spontaneous resolution has occurred by 3-6 months [7-8].

Based on clinical experience and practices in our medical center, we assumed the rate of early neuroimaging referral in older patients presenting with acute ocular motor mononeuropathy is greater when initially presented to a neurologist compared with an ophthalmologist. We thus conducted a retrospective study of patients 50 years of age and older with vasculopathic risk factors, presenting with acute isolated ocular motor nerve palsy, to demonstrate the approach differences between neurology and ophthalmology regarding the role of early neuroimaging. We compared the rate of early neuroimaging referrals between neurologists and ophthalmologists at initial presentation, and assessed the proportion of cases in which the final diagnosis (presumed microvascular ischemia versus another cause) changed after early neuroimaging.

\section{Methods}

Local institutional review board approval was obtained for this study, and the research adhered to the tenets of the Helsinki Declaration. Electronic hospital medical records were used to identify patients $\geq 50$ years of age, presenting with acute isolated third, fourth or sixth cranial nerve palsies, in our medical center, from January 2010 through December 2016. Isolated palsy was defined as the absence of other signs and symptoms, with the exception of headaches or periorbital pain within one month of the onset of diplopia. History of vasculopathic risk factors was obtained (i.e., Hypertension, hypercholesterolemia, diabetes mellitus, myocardial infarction, coronary artery disease, stroke and tobacco use) for each patient. Patients included in the study had at least one vasculopathic risk factor, because the population under debate regarding the role of early neuroimaging in acute isolated $4^{\text {th }}$ or $6^{\text {th }}$ nerve palsy is older individuals (age $\geq 50$ years) with vasculopathic risk factors, and thus patients with no vasculopathic risk factors were excluded.

Patients with systemic signs or localizing neurologic signs or symptoms suggesting a different cause for the isolated cranial nerve palsy were excluded from the study. Patients with a known history of myasthenia gravis, malignancy, head trauma, neurosurgical intervention, orbital disease, strabismus surgery, thyroid eye disease, temporal arteritis and multiple sclerosis were excluded as well.

Data were collected on demographics, history of vasculopathoc risk factors, neuroimaging results and comparison of imaging referral rates between neurologists and ophthalmologists at initial presentation. Electronic hospital medical records were used to assess the presence or absence of prior ocular motor palsy, neurologic disease, cancer or any other relevant history. Results of diagnostic testing (e.g., erythrocyte sedimentation rate, C-reactive protein, thyroid function tests, lumbar punctures and acetylcholine receptor antibody test,) were recorded.

Finally, after retrospective evaluation, the patients were divided by the cause of their ocular motor palsy to presumed microvascular ischemia versus another cause. A presumed microvascular cause was assigned for patients in whom early neuroimaging (MRI or contrast enhanced CT) and/or clinical testing did not reveal an alternative cause, other neurological signs remained absent, and the ophthalmoparesis resolved spontaneously.

The study's primary end point was comparing the rate of early neuroimaging referral between neurologists and ophthalmologists at initial presentation. The study's secondary end point was assessing the proportion of cases in which the final diagnosis (presumed microvascular ischemia versus another cause) has changed after early neuroimaging.

The statistical analysis was carried out using SPSS software, version 20. Double exponential distribution was performed with $\mathrm{p}<0.05$ considered statistically significant. Mean, standard deviation (SD), median and range were used to summarize the continuous variables. Two-sample t-tests were used to compare means of normally distributed variables, and Pearson chi square tests were used for categorical variables. Mann Whitney tests were used if data distribution did not follow a normal distribution.

\section{Results}

Out of 285 patients $\geq 50$ years of age presenting with acute diplopia from January 2010 through December 2016, 54 had vasculopathic risk factors and acute isolated ocular motor nerve palsy, and were included in the study. The remaining 231 patients met the exclusion criteria.

Of the 54 patients included, 16 patients had cranial nerve III palsy (twelve were partial and four were complete), 14 patients had cranial nerve IV palsy and 24 patients had cranial nerve VI palsy.

The mean $( \pm$ SD) age of the cohort was $69 \pm 9.8$ years (median 68.5 years, range 50-98 years). The mean ( \pm SD) duration of diplopia at presentation was $5.2 \pm 6.7$ days (median 3, range 1-30 days). The mean ( \pm SD) duration of follow-up was $21.12 \pm 27.88$ months (median six months, range 3-91 months). The cohort consisted of 33 men and 21 women. A comparison of patient characteristics between the patients with presumed microvascular ischemia versus other causes is shown in Table 1. Distribution of age and gender were similar between the two groups. The association of hypertension with presumed microvascular ischemia was most frequent in a univariate analysis, although this did not reach significance $(p=0.720)$. Sixth cranial nerve was most frequently involved, and had the highest incidence of other causes $(4 / 5,80 \%)$. 
Table 1. Characteristics of patients with acute isolated ocular motor nerve palsy of presumed microvascular ischemia versus other causes.

\begin{tabular}{llll}
\hline Characteristics & $\begin{array}{l}\text { Presumed microvascular } \\
\text { ischemia }(\boldsymbol{n}=\mathbf{4 9})(\mathbf{9 1 \% )}\end{array}$ & $\begin{array}{l}\text { Other causes } \\
(\boldsymbol{n}=\mathbf{5})(\mathbf{9 \%})\end{array}$ & $\begin{array}{l}\text { P- } \\
\text { value }\end{array}$ \\
\hline Age, Mean $( \pm$ SD) & $69( \pm 10.2)$ & $68.8( \pm 5.4)$ & $0.977 \mathrm{a}$ \\
Age, Median (range) & $68(50-98)$ & $69(63-76)$ & $0.639 \mathrm{~b}$ \\
Female & $19(39 \%)$ & $2(40 \%)$ & \\
Male & $30(61 \%)$ & $3(60 \%)$ & $0.957 \mathrm{c}$ \\
Nerve involved $(n)$ & $49(91 \%)$ & $5(9 \%)$ & \\
$3(16)$ & $15(31 \%)$ & $1(20 \%)$ & \\
$4(14)$ & $14(28 \%)$ & 0 & $0.205 \mathrm{c}$ \\
$6(24)$ & $20(41 \%)$ & $4(80 \%)$ & \\
\hline
\end{tabular}

${ }^{\mathrm{a} P}$-value from Mann Whitney test

${ }^{\mathrm{b}} \mathrm{P}$ - value from Median test

${ }^{\mathrm{c}} \mathrm{P}$-value from Pearson chi square test

$n=$ number of patients, SD-Standard deviation

Overall, neuroimaging and/or other diagnostic testing identified a cause other than presumed microvascular ischemia in $5 / 54$ patients $(9 \%)$, including two patients with myasthenia gravis (MG) and one patient each with pituitary macroadenoma, petroclival meningioma and brain metastasis from metastatic hepatocellular carcinoma (Table 2).

Table 2. Causes other than presumed microvascular ischemia for acute ocular motor mono-neuropathies in older patients.

\begin{tabular}{ll}
\hline Cranial nerve affected & Etiology \\
\hline Third & Pituitary macroadenoma \\
Sixth & Brain metastasis from metastatic \\
Sixth & hepatocellular carcinoma. \\
Sixth & Petroclival meningioma \\
\hline
\end{tabular}

* There were two patients with myasthenia gravis.

34 patients $(34 / 54,63 \%)$ were diagnosed with presumed microvascular palsy which spontaneously resolved within approximately three months, and 15/54 (28\%) patients did not return for a follow-up visit. $3 / 15$ patients passed away and $2 / 15$ could not be reached by phone. $10 / 15$ patients were contacted by phone and all reported their diplopia had resolved in approximately three months.

Overall, 28/54 (52\%) patients were initially seen by a neurologist and 26/54 (48\%) patients were initially seen by an ophthalmologist. 45 patients were referred to early neuroimaging (16 with $3^{\text {rd }}$ nerve palsy, 13 with $4^{\text {th }}$ nerve palsy and 16 with $6^{\text {th }}$ nerve palsy). Among them, 33/45 patients $(73 \%)$ were referred to contrast enhanced CT, six of whom were subsequently referred to MRI on follow-up due to suspected findings or partial resolution. Two were referred to early MRI, and one was referred to CT without contrast because of contrast sensitivity and was later referred to MRI. 9/45 (20\%) were referred to CT without contrast, all by neurologists.

In order to investigate the yield of early neuroimaging, the subset of patients analyzed were without third cranial nerve palsies, because there is a consensus regarding the necessity of early imaging in these patients. We found that the rate of patients referred to early neuroimaging was significantly greater when initially presented to a neurologist compared with an ophthalmologist. Out of 38 patients presented with isolated fourth or sixth cranial nerve palsies, 29 patients were referred to early neuroimaging (twenty patients were referred by a neurologist and nine by an ophthalmologist, $\mathrm{p}<0.001$ ). The remaining nine patients who were not referred to early neuroimaging were all initially presented to an ophthalmologist.

Only $4 / 38(10 \%)$ were found to have a cause other than presumed microvascular ischemia (Table 2). Two of them $(2 / 38,5 \%)$ had a causative lesion upon early neuroimaging. One was a patient with a history of hypertension and hypercholesterolemia, who presented with isolated right sixth cranial nerve palsy, and was found to have a petroclival meningioma with involvement of the right cavernous sinus, that was subsequently treated with stereotactic radiotherapy. The second was a patient with multiple vasculopathic risk factors, who presented with two weeks history of diplopia due to right sixth cranial nerve palsy. The MRI revealed a lytic lesion in the right petrous bone that was later diagnosed as brain metastasis from metastatic hepatocellular carcinoma.

None of the patients with isolated third nerve palsy $(n=16)$ were found to have a brain aneurysm. There was only one patient with third cranial nerve palsy who was found to have a cause other than presumed microvascular ischemia, with a causative lesion on MRI. The patient had multiple vasculopathic risk factors, and presented with three weeks history of diplopia due to complete right third nerve palsy. The MRI revealed a pituitary macro-adenoma with involvement of the right cavernous sinus, which was subsequently treated with endoscopic trans-sphenoidal surgery.

Additionally, in our study there were two patients with sixth cranial nerve palsies diagnosed with $\mathrm{MG}$ based on a positive acetylcholine receptor antibody test. These patients presented only with intermittent diplopia, and did not have any other systemic symptoms of MG. they were both treated with Pyridostigmine with resolution of their symptoms in approximately three months.

Assessment of the proportion of cases in which the final diagnosis (presumed microvascular ischemia versus another cause) has changed after early neuroimaging revealed that overall, $3 / 45$ patients $(7 \%)$ referred to early neuroimaging were found to have causative lesions, one with $3^{\text {rd }}$ nerve palsy and two with $6^{\text {th }}$ nerve palsy.

\section{Discussion}

As far as we know, our study is the first study demonstrating approach differences between neurologists and ophthalmologists regarding the role of early neuroimaging in this clinical setting. Our results show that the rate of patients referred to early neuroimaging was significantly greater when initially presented to a neurologist compared with an ophthalmologist. Possible reasons for the observed referral differences could be due to differences in clinical background and experience. Ophthalmologists follow neuroophthalmologic guidelines regarding acute isolated ocular 
motor nerve palsies, whereas neurologists usually treat patients with cerebrovascular accidents and thus tend to refer most patients to early neuroimaging.

In the current retrospective chart review, the yield of early neuroimaging in older vasculopathic patients with acute 4th or 6th cranial nerve palsies was low. In this group of patients ( $n=38$ ), early neuroimaging was obtained in $29 / 38$ patients, and we identified only two subjects with a causative lesion found from early neuroimaging (2/29, 7\%). 22/38 patients (58\%) were diagnosed with microvascular ischemia and had spontaneous resolution in approximately three months. After Exclusion of patients with third nerve palsies, there was approximately 1 in a 15 chance that a patient with vasculopathic risk factors had palsy due to a nonmicrovascular cause, as similar to what was found in a previous study [1].

According to current accepted guidelines regarding neuroimaging recommendations [7], patients should receive an MRI in the setting of an acute isolated ocular motor nerve palsy if any of the following criteria are met: (1) age $<50$ years, (2) third nerve palsy, (3) history of cancer, (4) neurologic signs or symptoms. If neuroimaging is initially deferred, a lack of resolution by three months or failure to demonstrate any recovery by one month should prompt further investigations, including neuroimaging [3-4].

Early neuroimaging in older vasculopathic patients remains controversial for several reasons, such as costly expenses and presumed low yield, as well as a belief that delaying neuroimaging does not necessarily change the prognosis. Three prior prospective studies have addressed this question [5-7]. In one study [5], the yield of MRI in identifying other causes for sixth nerve palsy was $15 \%$ (four patients, two with a metastasis, one with a meningioma and one with an aneurysm). The median age of the cohort in this study was 43 years, an age where there is a higher likelihood of finding a specific cause [5]. Another study [6] found a $13.6 \%(9 / 66)$ incidence of other causes in patients presenting with acute ocular motor mononeuropathies. The incidence of other causes for fourth and sixth nerve palsies was $13.5 \%$ $(5 / 37)$, including neoplasm, brainstem infarction, demyelinating disease and pituitary apoplexy. The authors concluded early neuroimaging should be performed in all patients [6]. On the other hand, a third study [7] investigated the cost utility and yield of neuroimaging for acute ocular motor mononeuropathies in adults $>50$ years, and found a low prevalence of causative lesions on MRI $(1.1 \%, 1 / 93$ patients) [7]. The one patient identified presented with a sixth nerve palsy due to a pontine hemorrhage, and did not need any intervention. The authors concluded that early neuroimaging was not cost-effective in older adults with the presence of vasculopathic risk factors. The above two studies [6-7] were single center studies, and all patients with a history of cancer, head trauma or neurologic and orbital diseases were excluded, similar to our study.

A recent multicenter prospective study [1], assessed the yield of neuroimaging in 109 patients presenting with acute third, fourth or sixth nerve palsy, and found the overall yield of neuroimaging to be $16.5 \%$. In patients with fourth and sixth nerve palsy with vasculopathic risk factors alone and no significant past medical history, the yield was found to be only $4.6 \%$. The causes included stroke, neoplasm, inflammation and pituitary apoplexy. The authors of this large study concluded that a contrast-enhanced brain MRI play an important role in the initial evaluation of patients who present with ocular motor mononeuropathies, including patients over 50 years of age [1].

In our study it was debatable whether or not early neuroimaging would have changed the prognosis in cases with a cause other than presumed microvascular ischemia. As stated, 2/38 patients with $4^{\text {th }}$ or $6^{\text {th }}$ nerve palsies had a causative lesion found on early neuroimaging. One patient presented with right $6^{\text {th }}$ cranial nerve palsy and was diagnosed with petroclival meningioma (Figure 1). A delay in diagnosis during an observation period would likely have had no impact on the final treatment or prognosis for this patient. The other patient presented with right $6^{\text {th }}$ cranial nerve palsy and was ultimately diagnosed with brain metastasis (Figure 2) from metastatic hepatocellular carcinoma involving multiple organs. Unfortunately, this patient died seven months later.

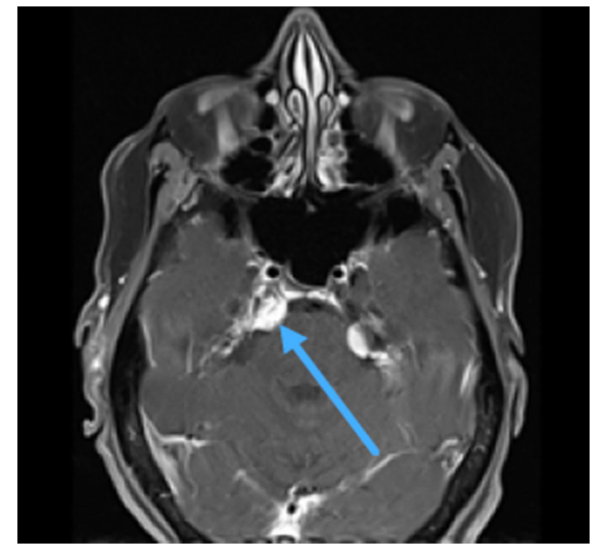

Figure 1. Patient with acute isolated right $6^{\text {th }}$ cranial nerve palsy. Axial T1 MRI with contrast showing several meningiomas, including right petroclival meningioma with involvement of right cavernous sinus.

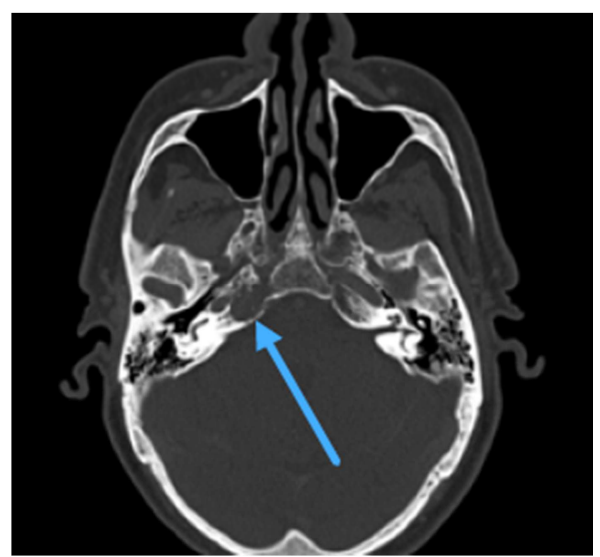

Figure 2. Patient with acute isolated right $6^{\text {th }}$ cranial nerve palsy. Head CT with bone window showing expansile lytic lesion in right petrous apex, in a patient with metastatic hepatocellular carcinoma, who presented with acute isolated right $6^{\text {th }}$ cranial nerve palsy. 
Moreover, prognosis-modifying etiologies, such as brain aneurysms, were not present in all 54 patients in our study, or in patients of previous studies [1, 6-7]. However, in the group of patients with $3^{\text {rd }}$ nerve palsy $(n=16)$, early neuroimaging did change management and prognosis in one patient, in whom a causative lesion was found (Figure 3).
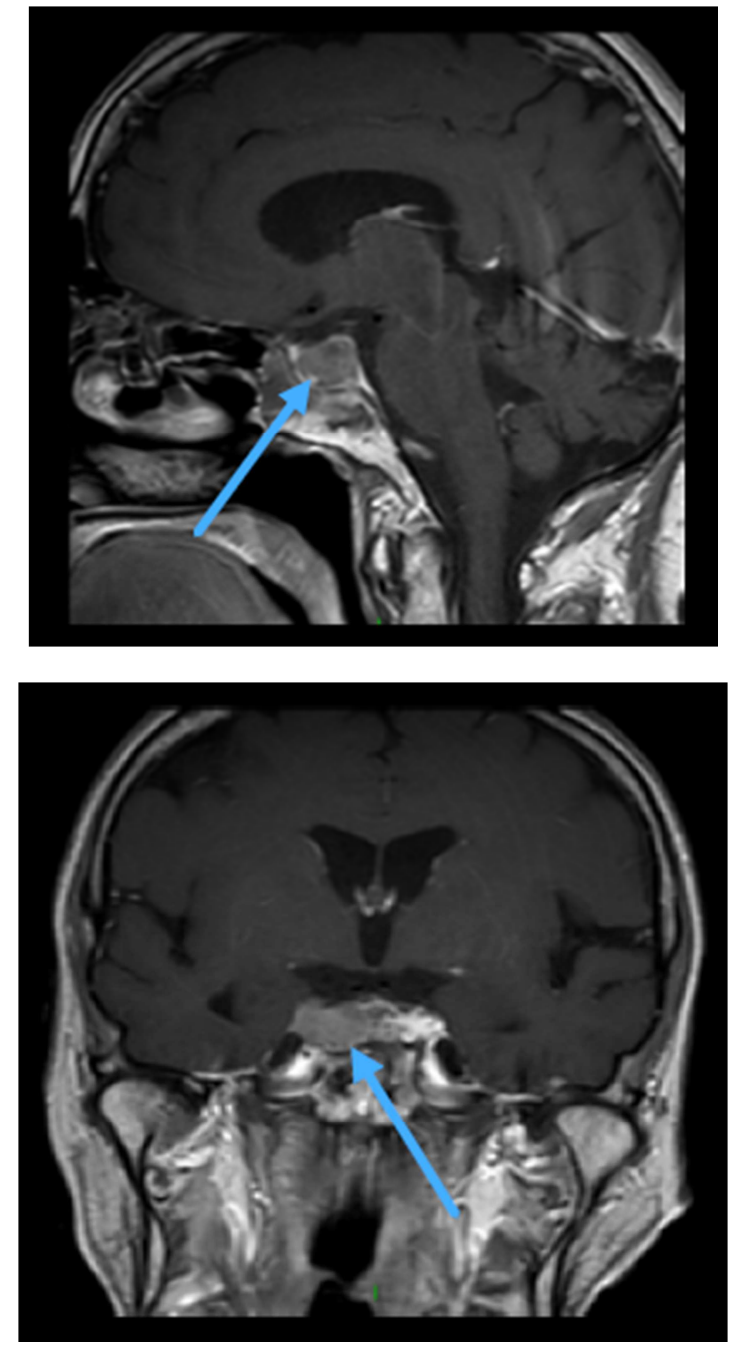

Figure 3. Patient with acute isolated right $3^{\text {rd }}$ cranial nerve palsy. (Left) Sagittal and (right) Coronal T1 MRI with contrast showing pituitary macroadenoma in a patient with acute isolated right $3^{\text {rd }}$ cranial nerve palsy.

Our single-center study is limited by its small size, its retrospective-cohort and non-randomized nature. There is a lack of large-scale prospective age-specific studies to address the diagnostic yield of early neuroimaging in older adults presenting with acute ocular motor mononeuropathies.

\section{Conclusions}

The role of early neuroimaging in older patients with vascular risk factors presenting with acute isolated $4^{\text {th }}$ or $6^{\text {th }}$ cranial nerve palsies is still debatable. Previous studies have found the yield of early neuroimaging to be $1-15 \%$, similar to our study (7\%). Despite costly expenses and presumed low yield, and even though performing early neuroimaging does not necessarily change the prognosis, it is advised to make the decision individually based on thorough history taking and physical examination findings, as performing early neuroimaging can change the clinical management in certain cases.

The decision to perform early neuroimaging in this clinical setting should be weighed against observation alone, and should be obtained if there is a lack of resolution by three months or failure to demonstrate any recovery by one month.

We emphasize the importance of thorough history taking and prudent physical examinations, as well as close collaboration between neurologists and ophthalmologists, in identifying patients at higher risk for serious underlying pathologies and thus in need of additional work-up, since those are the patients with the highest yield for early neuroimaging.

\section{Acknowledgements}

No Financial disclosures.

The authors declare that they have no competing interests.

Our study has been previously presented as a conference abstract and accepted for poster presentation at NANOS Annual Meeting, March 2018, Waikoloa Village, Hawaii, USA.

\section{References}

[1] Tamhankar MA, Biousse V, Ying GS, et al. Isolated third, fourth, and sixth cranial nerve palsies from presumed microvascular versus other causes: a prospective study. Ophthalmology 2013 Nov; 120 (11): 2264-9.

[2] Tamhankar MA, Volpe NJ. Management of acute cranial nerve 3,4 and 6 palsies: role of neuroimaging. Curr Opin Ophthalmol. 2015 Nov; 26 (6): 464-8.

[3] Kung NH, Van Stavern GP. Isolated ocular motor nerve palsies. Semin Neurol. 2015 Oct; 35 (5): 539-48.

[4] Volpe NJ. Lee AG. Do patients with neurologically isolated ocular motor cranial nerve palsies require prompt neuroimaging? J Neuroophthalmol. 2014 Sep; 34 (3): 301-5.

[5] Bendszus M, Beck A, Koltzenburg M, et al. MRI in isolated sixth nerve palsies. Neuroradiology 2001; 43: 742-5.

[6] Chou KL, Galetta SL, Liu GT, et al. Acute ocular motor mononeuropathies: prospective study of the roles of neuroimaging and clinical assessment. J Neurol Sci 2004; 219: 35-9.

[7] Murchison AP, Gilbert ME, Savino PJ. Neuroimaging and acute ocular motor mononeuropathies: a prospective study. Arch Ophthalmol. 2011; 129: 301-5.

[8] Chi SL, Bhatti MT. The diagnostic dilemma of neuro-imaging in acute isolated sixth nerve palsy. Curr Opin Ophthalmol. 2009; 20: 423-9.

[9] Panda BB, Parija S, Mallick J, Pujahari S. Oculomotor Nerve Palsy as a Rare Presentation and First Sign of Multiple Myeloma. J Clin Diagn Res. 2016 May; 10 (5). 
[10] Shechtman DL, Woods AD, Tyler JA. Pupil sparing incomplete third nerve palsy secondary to a cavernous sinus meningioma: challenges in management. Clin Exp Optom. 2007 Mar; 90 (2): 132-8.

[11] Muthukrishnan J, Bharadwaj K, Singh Y. Isolated fourth cranial nerve palsy due to pituitary macroadenoma. Med J Armed Forces India. 2016 Dec; 72 (Suppl 1): S67-S69.

[12] Mallery RM, Klein JP, Pless ML. Isolated sixth nerve palsy from hemorrhage of a pontine cavernous malformation. $\mathrm{J}$ Neuroophthalmol. 2012 Dec; 32 (4): 335-7.

[13] Ogawa K, Suzuki Y, Takahashi K, Kamei S, Ishikawa H. Clinical Study of Eleven Patients with Midbrain InfarctionInduced Oculomotor Nerve Palsy. J Stroke Cerebrovasc Dis. 2016 Jul; 25 (7): 1631-1638.

[14] Lai G, Rodriguez MI, Scumpia AJ. Oculomotor Nerve Palsy Secondary to Cavernous Internal Carotid Aneurysm. Clin Pract Cases Emerg Med. 2018 Jan 9; 2 (1): 93-94.

[15] Lavin PJ, Aulino JM, Uskavitch D. "Ophthalmoplegic migraine" with reversible MRI enhancement of the cisternal sixth cranial nerve. J Neuroophthalmol. 2009 Jun; 29 (2): 1513 .

[16] Jo YS, Kim SK, Kim DH, Kim JH, Na SJ. Complete Oculomotor Nerve Palsy Caused by Direct Compression of the Posterior Cerebral Artery. J Stroke Cerebrovasc Dis. 2015 Jul; 24 (7): e189-90.

[17] Tan T, Tee JW, Wang YY. Oculomotor nerve palsy secondary to aberrant posterior cerebral artery. BMJ Case Rep. 2014 Jun $30 ; 2014$.

[18] Tokunaga M, Fukunaga K, Nakanishi R, Watanabe S, Yamanaga $\mathrm{H}$. Midbrain infarction causing oculomotor nerve palsy and ipsilateral cerebellar ataxia. Intern Med. 2014; 53 (18): 2143-7.

[19] Zadro I, Barun B, Habek M, Brinar VV. Isolated cranial nerve palsies in multiple sclerosis. Clin Neurol Neurosurg. 2008 Nov; 110 (9): 886-8.

[20] Wu HC, Ro LS, Chen CJ, Chen ST, Lee TH, Chen YC, Chen CM. Isolated ocular motor nerve palsy in dural carotidcavernous sinus fistula. Eur J Neurol. 2006 Nov; 13 (11): $1221-5$

[21] Chang JH, Shin YB, Sohn HJ, Ko HY, Lee TH, Ha YH. Isolated bilateral abducens nerves palsy due to traumatic delicate arteriovenous fistula. Brain Inj. 2010 Jan; 24 (1): 469.

[22] Lee SH, Park SW, Kim BC, Kim MK, Cho KH, Kim JS.
Isolated trochlear palsy due to midbrain stroke. Clin Neurol Neurosurg. 2010 Jan; 112 (1): 68-71.

[23] Raghavendra S, Vasudha K, Shankar SR. Isolated trochlear nerve palsy with midbrain hemorrhage. Indian J Ophthalmol. 2010 Jan-Feb; 58 (1): 66-7.

[24] Ashker L, Weinstein JM, Dias M, Kanev P, Nguyen D, Bonsall DJ. Arachnoid cyst causing third cranial nerve palsy manifesting as isolated internal ophthalmoplegia and iris cholinergic supersensitivity. J Neuroophthalmol. 2008 Sep; 28 (3): 192-7.

[25] Duma SR, Ghattas S, Chang FCF. Internal Carotid Artery Occlusion Causing Acute Cranial Neuropathies. J Stroke Cerebrovasc Dis. 2019 Apr; 28 (4): e5-e6.

[26] Watanabe A, Horikoshi T, Uchida M, Kinouchi H. Internal carotid artery occlusion manifesting only as oculomotor nerve palsy. J Stroke Cerebrovasc Dis. 2008 Nov-Dec; 17 (6): 4335 .

[27] Lee CY, Chen HC, Lin HY, Kao LY. Oculomotor palsy as a single presenting sign of midbrain hemorrhage. Taiwan $\mathrm{J}$ Ophthalmol. 2018 Apr-Jun; 8 (2): 108-110.

[28] Yokosako S, Kikkawa Y, Takeda R, Ikeda T, Kurita H. Oculomotor Nerve Palsy in a Patient with a Ruptured Middle Cerebral Artery Aneurysm. J Med Invest. 2017; 64 (1.2): 165167.

[29] Somagawa C, Fukuda Y, Yoshimura S, Satoh K, Hiu T, Ono T, Ushijima R, Toda K, Tsutsumi K. A Rare Case of Ruptured Internal Carotid-Posterior Communicating Artery Aneurysm Associated with Contralateral Delayed Oculomotor Nerve Palsy. No Shinkei Geka. 2017 Jul; 45 (7): 629-635.

[30] Cho WJ, Joo SP, Kim TS, Seo BR. Pituitary apoplexy presenting as isolated third cranial nerve palsy with ptosis: two case reports. J Korean Neurosurg Soc. 2009; 45:118-21. [PubMed: 19274125].

[31] Zoli M, Mazzatenta D, Pasquini E, Ambrosetto P, Frank G. Cavernous sinus apoplexy presenting isolated sixth cranial nerve palsy: case report. Pituitary. 2012 Dec; 15 Suppl 1: S3740.

[32] Bahmani Kashkouli M, Khalatbari MR, Yahyavi ST, BorgheiRazavi H, Soltan-Sanjari M. Pituitary apoplexy presenting as acute painful isolated unilateral third cranial nerve palsy. Arch Iran Med. 2008 Jul; 11 (4): 466-8.

[33] Warwar RE, Bhullar SS, Pelstring RJ, Fadell RJ. Sudden death from pituitary apoplexy in a patient presenting with an isolated sixth cranial nerve palsy. J Neuroophthalmol. 2006; 26: 95-7. [PubMed: 16845307]. 